\title{
Temporal coherence shaping based on spectral-domain destructive interference of pulses with different self-phase modulations
}

D. Wei

weidong@nanolab.t.u-tokyo.ac.jp

\section{K. Takamasu}

H. Matsumoto
Global Center of Excellence Program - Mechanical Systems Innovation-, School of Engineering, The University of Tokyo, Hongo 7-3-1, Bunkyo-ku, Tokyo 113-8656, Japan

Department of Precision Engineering, The University of Tokyo, Hongo 7-3-1, Bunkyo-ku, Tokyo 1138656, Japan

Department of Precision Engineering, The University of Tokyo, Hongo 7-3-1, Bunkyo-ku, Tokyo 1138656, Japan

We show via a numerical simulation that the temporal coherence function (TCF) can be shaped by the destructive interference of pulses characterized by different amounts of self-phase modulation (SPM) in the spectral domain. We find that pulse spectra destructively interfering with one another can yield a TCF with distinct peaks. Numerical investigation demonstrates that the shape of the TCF is changeable not only by broadening the spectrum but also by overlapping spectra of the pulses that have experienced different amounts of SPM. [DOI: http://dx.doi.org/10.2971/jeos.2013.13018]

Keywords: Temporal coherence function, self-phase modulation, destructive interference, shaping, spectra

\section{INTRODUCTION}

Self-phase modulation (SPM) has attracted considerable attention because of its potential for applications such as soliton-based optical communication [1], temporal pulse compression [2], and fast optical switching [3]. SPM is a nonlinear phase delay that is proportional to the intensity of the pulse, and which occurs when a pulse propagates through a medium with the optical Kerr effect (e.g., an optical fiber). The first demonstration of the generation of SPM, in a $\mathrm{CS}_{2}$-filled cell, was reported in 1967; since then, SPM has been reported in a variety of media with the optical Kerr effect [4].

SPM has been used to contribute spectral broadening to supercontinuum generation by introducing a higher-order maximum nonlinear phase shift [2]. For example, it has been reported that strong SPM can be used to generate a 4-ps pulse [5]. A supercontinuum light source was used in a Michelson interferometer as a primary means of improving the axial resolution in optical coherence tomography. In a Michelson interferometer with a white light source, a one-to-one relationship arises between the heights of a reflecting surface and the peaks of the fringe-visibility curve. Based on the WienerKhintchine theorem, we can increase the sharpness of the peak of the fringe-visibility curve by broadening the spectrum of the source, due to their Fourier relationship. A narrow peak is required because, if two neighboring peaks are sufficiently wide that they can overlap, the axial resolution of position will be poor. To improve the axial resolution, a spectrally broadened light source is required. However, the use of SPM to control the properties of light sources has been largely restricted to spectral broadening, although SPM is likely to be useful for other optical applications.
Theoretically, if the incoming laser pulse is symmetric, then the frequency distribution of the SPM is also expected to be symmetric, and the output spectrum should show broadening and a quasi-periodic shape. The output spectrum can be classified by the maximum phase shift $\phi_{\max }=(M-1 / 2) \pi$, where $M$ is the number of spectral peaks. The spectral evolution resulting from changing the power of an incoming pulse has been experimentally observed [6]. The proposed method was developed based on the idea of Fourier series expansion (Figure 1). As is well known, a Fourier series expansion represents an arbitrary periodic function as a sum of a series of trigonometric functions. Herein, we consider the possibility of thinking that SPM is capable of generating a set of basic (quasi-periodic) frequency-domain modes. These modes are spectra with different maximum phase shifts. By using these modes, we can synthesize a spectrum that differs from the spectrum that can be obtained by applying spectral broadening to a single mode-locked spectrum. We note that these modes are not orthogonal to each other and that such orthogonality is not required in this application.

In this work, we present the result of a numerical simulation to shape the temporal coherence function (TCF) by destructive overlapping of the spectra of different self-phasemodulated Gaussian pulses obtained by propagating through different lengths of nonlinear fibers. Coherence shaping by synthesis of light-emitting diodes [7], by optimal spectral reshaping of a single light source [8], and by a frequencymodulation technique [9] have been reported. Recently, spatial coherence function control using the spatial distribution of a single-wavelength light source has also been proposed, demonstrated, and applied [10]-[12]. The application of tem- 


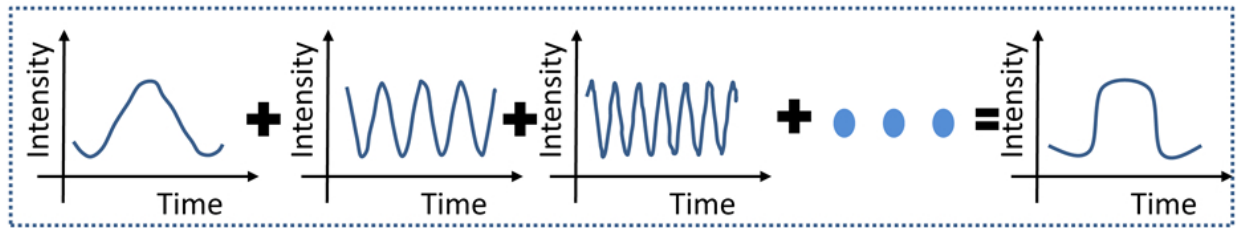

(a)

Construction of a temporal coherence function using superposition of SPM modes

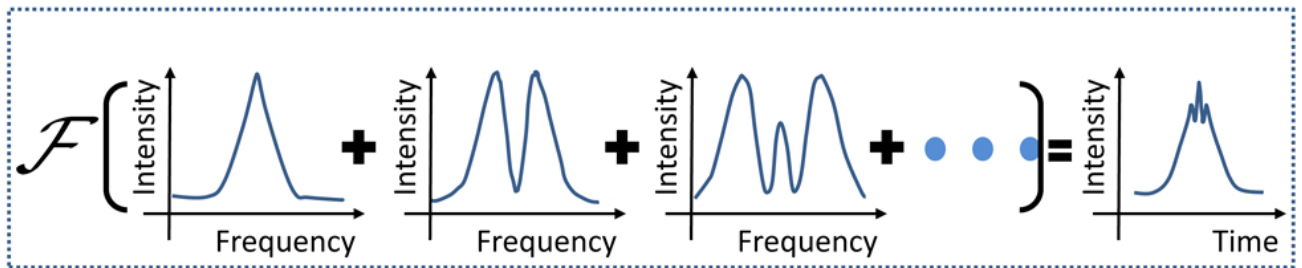

(b)

FIG. 1 Analogy between a Fourier series expansion and the proposed method. (a) Simplified schematic of a Fourier series expansion. (b) Simplified construction of a TCF using superposition of SPM modes. The character $\mathcal{F}$ denotes the Fourier transform.

poral coherence function synthesis can be found in Refs. [13]-[15]. To the best of our knowledge, very few studies have been carried on the nonlinear means that can be used to shape the TCF, and such nonlinear methods are likely to be more useful for functional metrology applications.

\section{PRINCIPLES OF METHOD DESIGN}

To explain our scheme, we begin with a general treatment of SPM, a more detailed version of which can be found in Ref. [4]. The general solution of the field amplitude of SPM can be obtained as [4]

$$
E(l, t)=E(0, t) \exp \left[i|E(0, t)|^{2}\left(l / l_{N L}\right)\right],
$$

where $l$ is the fiber length, $E(0, t)$ is the field of the initial pulse at $l=0$, and $l_{N L}$ is the effective length of the nonlinear fiber. The length $l_{N L}$ is related to the peak power of the initial pulse and the nonlinear-index parameters. Using the Fourier transform, the shape of the power spectrum $S(l, f)$, which is spectrally broadened by SPM, is given by

$$
S(l, f)=<|\tilde{E}(l, f)|^{2}>=<|\mathcal{F}[E(l, t)]|^{2}>.
$$

Here, the character $\mathcal{F}$ represents the Fourier transform. $<>$ denotes ensemble averaging.

In a Michelson interferometer, the interferometric signal $\Gamma(l, t)$ is given by the inverse Fourier transform of Eq. (2):

$$
\Gamma(l, t)=\mathcal{F}^{-1}[S(l, f)]
$$

The envelope of the interference fringes can be written as:

$$
\gamma(l, t)=\Gamma(l, t) / \Gamma(l, 0) \propto \mathcal{F}^{-1}\left\{<|\mathcal{F}[E(l, t)]|^{2}>\right\},
$$

Here, $\Gamma(l, 0)=<|E(l, 0)|^{2}>$ is a constant.

Let us consider the TCF formed by a Michelson interferometer. As shown in Figure 1(b), when the incident light on the Michelson interferometer is a set of pulses that have experienced different amounts of SPM, the total power spectrum is a superposition of the corresponding spectra with different amounts of SPM-induced broadening, which can be written as a superposition of terms of the form of Eq. (4). For the final envelope of the interference fringes, we obtain the expression

$$
\gamma_{M u l t i}(l, t) \propto \mathcal{F}^{-1}\left\{<\left|\sum_{q} \mathcal{F}\left[E_{q}(l, t)\right]\right|^{2}>\right\}
$$

where $q$ denotes the total number of overlapped pulses. By using the formula of the multinomial theorem, we can expand Eq. (5) to

$$
\begin{aligned}
& \gamma_{\text {Multi }}(l, t) \propto \mathcal{F}^{-1}\left\{\cdots+<\left|\mathcal{F}\left[E_{m}(l, t)\right]\right|^{2}>\right. \\
& \quad+<\left|\mathcal{F}\left[E_{n}(l, t)\right]\right|^{2}>+2<\mathcal{F}\left[E_{m}(l, t)\right] \mathcal{F}^{*}\left[E_{n}(l, t)\right]> \\
& \quad+\cdots\}
\end{aligned}
$$

Here, $<\left|\mathcal{F}\left[E_{m}(l, t)\right]\right|^{2}>$ and $<\left|\mathcal{F}\left[E_{n}(l, t)\right]\right|^{2}>$ are the $m$ th and the $n$th pulse spectra for different SPM values, respectively. Considering the cross-product of the different SPMbroadened spectra, $<\mathcal{F}\left[E_{m}(l, t)\right] \mathcal{F}^{*}\left[E_{n}(l, t)\right]>$, introduced us to a new and interesting effect. If there is a $\pi$ phase difference between $\mathcal{F}\left[E_{m}(l, t)\right]$ and $\mathcal{F}^{*}\left[E_{n}(l, t)\right]$, the two pulses will either partially or completely cancel each other out depending on the amount of overlap in the spectral domain. The fact that this destructive interference between $\mathcal{F}\left[E_{m}(l, t)\right]$ and $\mathcal{F}^{*}\left[E_{n}(l, t)\right]$ can change the shape of the TCF is verified in the following simulation.

\section{NUMERAL SIMULATIONS}

Modeling of the SPM is performed using the nonlinear Schrödinger equation [4]. Generally, it is not possible to solve the nonlinear Schrödinger equation analytically. Therefore, a large number of numerical methods have been developed to solve the nonlinear Schrödinger equation to understand various nonlinear effects (such as SPM, four-wave mixing, 

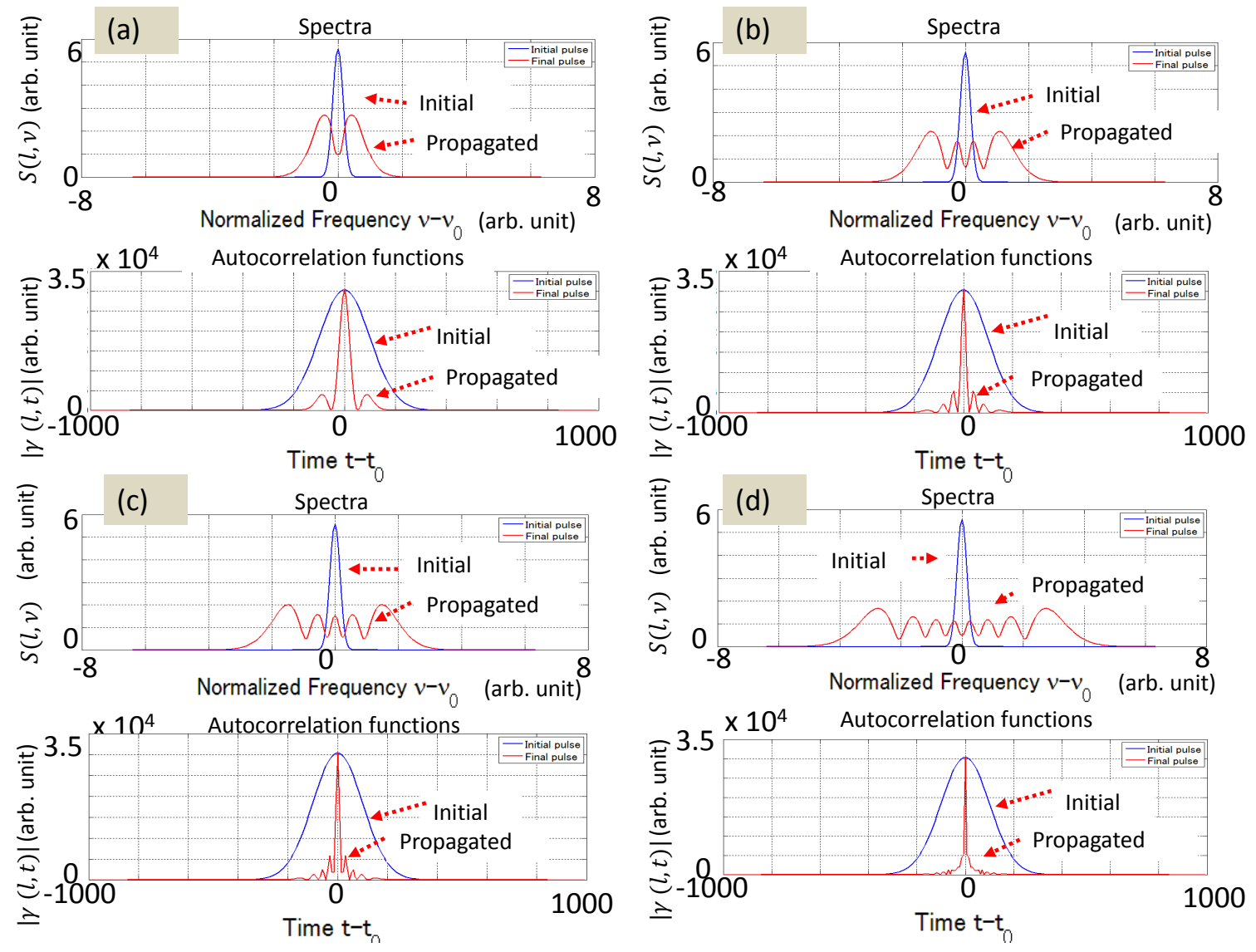

FIG. 2 Calculated forms of each of the basic spectral modes of SPM and the related autocorrelations. (a) The maximum phase shift is $2.5 \pi$. (b) The maximum phase shift is $6.5 \pi$. (c) The maximum phase shift is $8.5 \pi$. (d) The maximum phase shift is $14.5 \pi$.

and stimulated Raman scattering) that occur during pulse propagation. The most commonly used numerical algorithm is the split-step Fourier method (SSFM) because of its good performance, considering the calculation accuracy achieved for a given computing cost [16].

Here, we briefly introduce the SSFM. When the pulse spreads in the nonlinear material it experiences linear effects (dispersion, absorption, scattering loss, etc.) and nonlinear effects at the same time. The difficulty in solving a nonlinear Schrödinger equation lies in the difficulty of treating the interaction between linear and nonlinear mechanisms. The SSFM approaches this problem using the following idea. First, the pulse spreading distance in the nonlinear material is divided into small steps. Next, linear effects and nonlinear effects in each step are calculated separately in the order of the steps; the result is given as an input to the next step. The same calculation continues up to the last step. A more detailed explanation of the SSFM can be found in Ref. [4].

On the basis of Eq. (1), we use our simulation to compute each of the basic modes (namely, spectra with different maximum phase-shift values) as follows. Because the experimental situation is as ideal as the simple theory describes, we choose to simulate the SPM of a laser pulse transmitted through an optical fiber. We selected a symmetric Gaussian-shaped source with center wavelength $\lambda=1560 \mathrm{~nm}$ as our light source. The pulse width and peak power were set at $100 \mathrm{fs}$ and $0.03 \mathrm{~W}$, respectively. The nonlinear coefficient of the fiber was set to be $20 \mathrm{~W}^{-1} \mathrm{~km}^{-1}$. The number of small steps for SSFM was selected to be 110 .

We used a MATLAB-based program to realize the SSFM method for finding a series of basic modes of SPM. Though research on optimization of the calculation of the SSFM has been reported [17], because the computational complexity is small, the number of steps that the fiber is divided into and other such parameters have not been optimized. In the simulation, we are not considering the other parameters (for example, self-steepening, pulse intensity variation, and dispersion) that affect spectral broadening. The calculated form of SPM (see Figure 2) is in good agreement with previous experiments and calculations $[6,18]$, confirming our calculation.

We describe the calculation of the overlapping of spectra with different maximum phase shifts based on Eq. (5). We investigated the interaction between different SPM modes using our simulation. The maximum phase shift used in our simulation was limited by the capacity of the computer that we used.

Figure 3 shows the result of shaping of a TCF by combining the spectra of two pulses, one of which experienced some selfphase modulation. Because of the destructive interference between the two spectra, the distance between the first sidelobe and the central peak becomes larger. As noted in Figure 3(b), this change of the spectra makes the sidelobe of the TCF move away from the central peak of the TCF.

Figure 4 shows the result of shaping of a TCF by combin- 


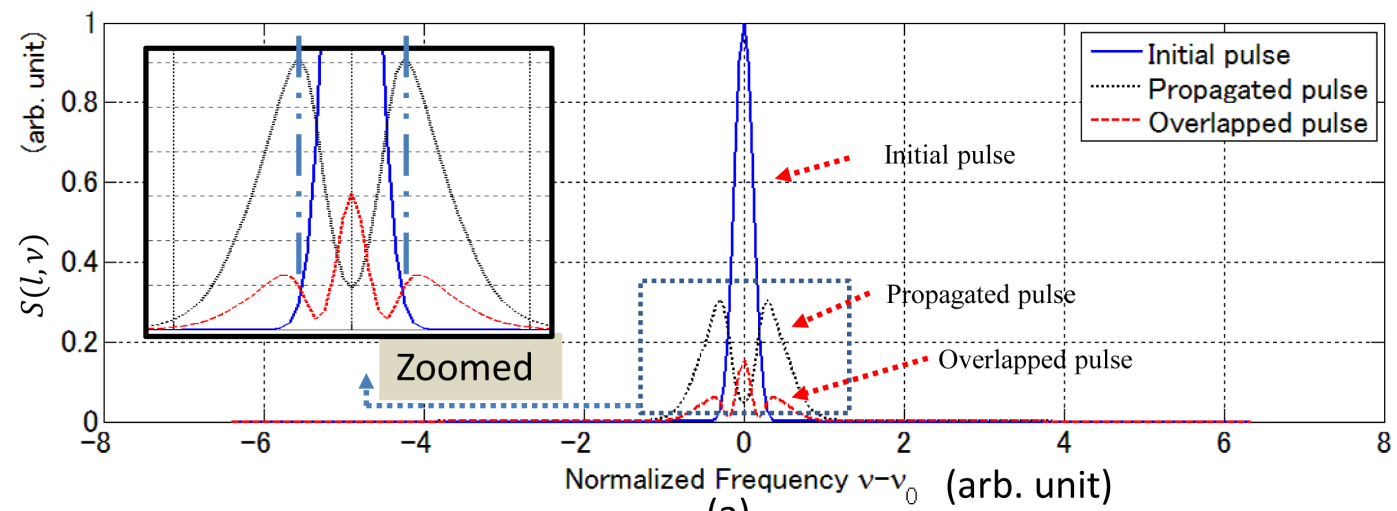

(a)

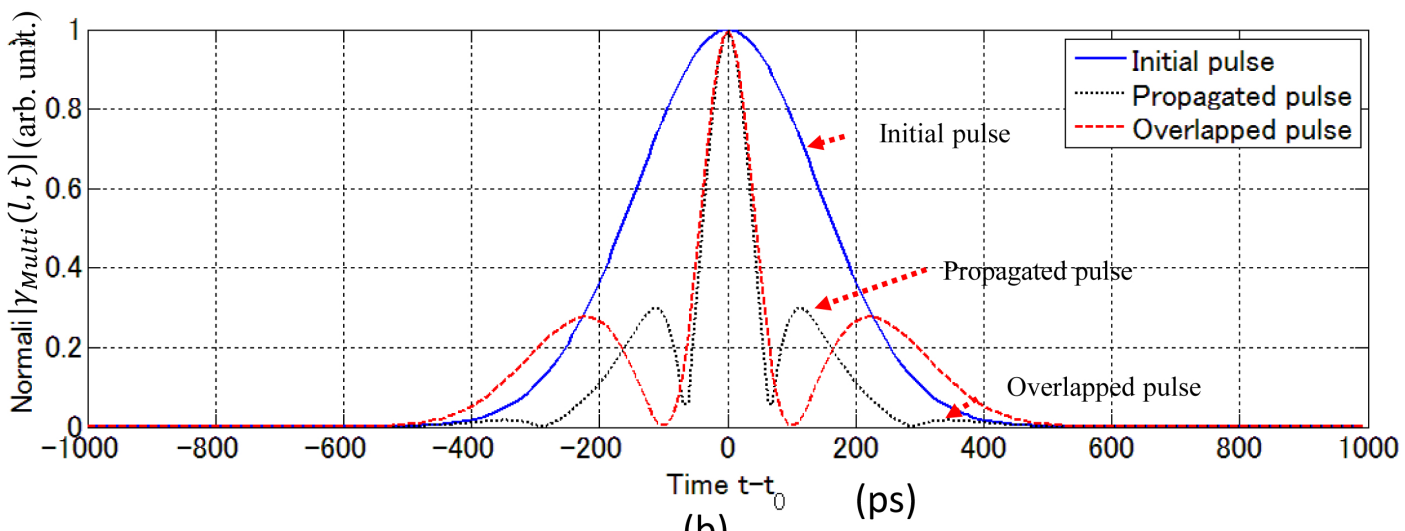

(b)

FIG. 3 Spectra and related autocorrelations. (a) Spectra of the initial pulse, the result of propagating the initial pulse, and the result of overlapping the initial pulse and the propagated pulse. The inset shows that the sidelobe peaks of the spectrum of the combined pulse shift away from the central peak due to destructive interference between the two spectra. The long dashed double-dotted line shows the peaks of the spectra of the propagated pulse. (b) Autocorrelations of the initial pulse, propagated pulse, and overlapped pulses. The ratio of the intensity of the pulses is $1: 1$. The maximum phase shifts of the two pulses are 0 and $2.5 \pi$, respectively.

ing the spectra of three pulses, two of which experienced self-phase modulation, in differing amounts. We chose the amounts of SPM based on the following idea. First, we needed a spectrum without SPM to introduce the central peak of the final spectrum. Second, two spectra that have experienced SPM are required to partially cancel their peaks near the center of the spectrum. As shown in Figure 4(a), the final spectrum has a central peak and relatively separated sidelobes. We can confirm that due to the interference among the three spectra, the correlation function has transformed into discrete peaks.

Here, we provide further explanation of the simulation. In this simulation, we tried all combinations of different SPM spectra. We chose as representative figures Figure 3 and Figure 4 to demonstrate the feasibility of TCF shaping based on the spectral domain destructive interference of pulses that have experienced different amounts of SPM. Figures 3 and 4 are not the only possible cases. The simulation results show that each of the shapes of the TCF resulting from the various combinations that we tried was distinct. This is because each SPM series has a different shape. If the amount of SPM is shifted from the selected value (namely, the value that we used in Figure 3 and Figure 4) but the spectra still maintain a relationship of destructive interference, the central peak and side lobes will be separated. If the spectra are no longer able to maintain the relationship of destructive interference, the central peak and side lobes will be merged together. Each of the spectra in the series has a possibly constructive or destructive interference relationship with every other spectrum in the series. Consequently, the shape of the TCF has a complicated dependence upon these intensities and phases, which is not easily summarized. The details are still under investigation. We believe that if one uses an optimization method with an objective function (for example, a maximum distance between the first sidelobe and the central peak of the spectrum of the TCF), this technique can be effectively used with relative ease of control.

We found that we can change the shape of the TCF by superposing the spectra of pulses that have accumulated different amounts of SPM. We confirmed via numerical simulation that the shape of the temporal coherence is changeable not only by broadening the spectrum, but also by overlapping spectra of the pulses that experienced different amounts of SPM. To change the shape of the TCF, we need to select the lengths of the fibers through which the pulses propagate and the specific values of the pulses' power. As is apparent, a single optical pulse propagating through the fiber causes various nonlinear phenomena. The spectrum of the pulse that undergoes such a nonlinear phenomenon becomes quite complex. In addition, further work is needed on the analytical expression for time coherence shaping. Finally, we emphasize that our approach is more general than the method of just broadening the spectrum of the source to get essentially a modified TCF peak.

We note that the combination of a multiple-arm Michelson interferometer [19] and a spatial spectral interferometry [20] is a promising scheme for experimental demonstrations of the 


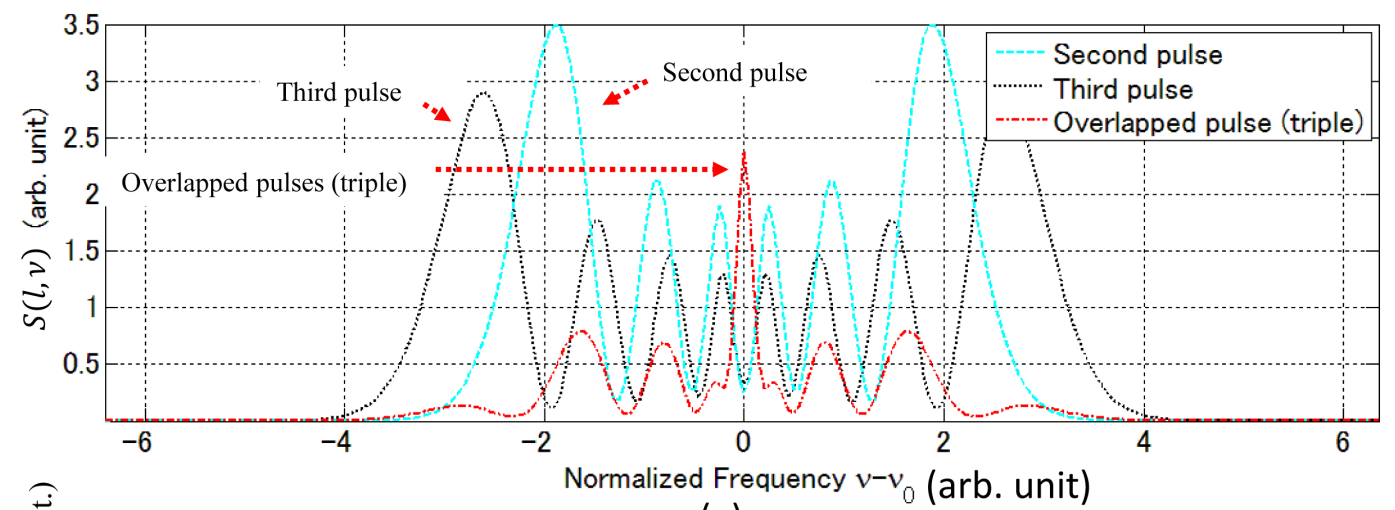

(a)

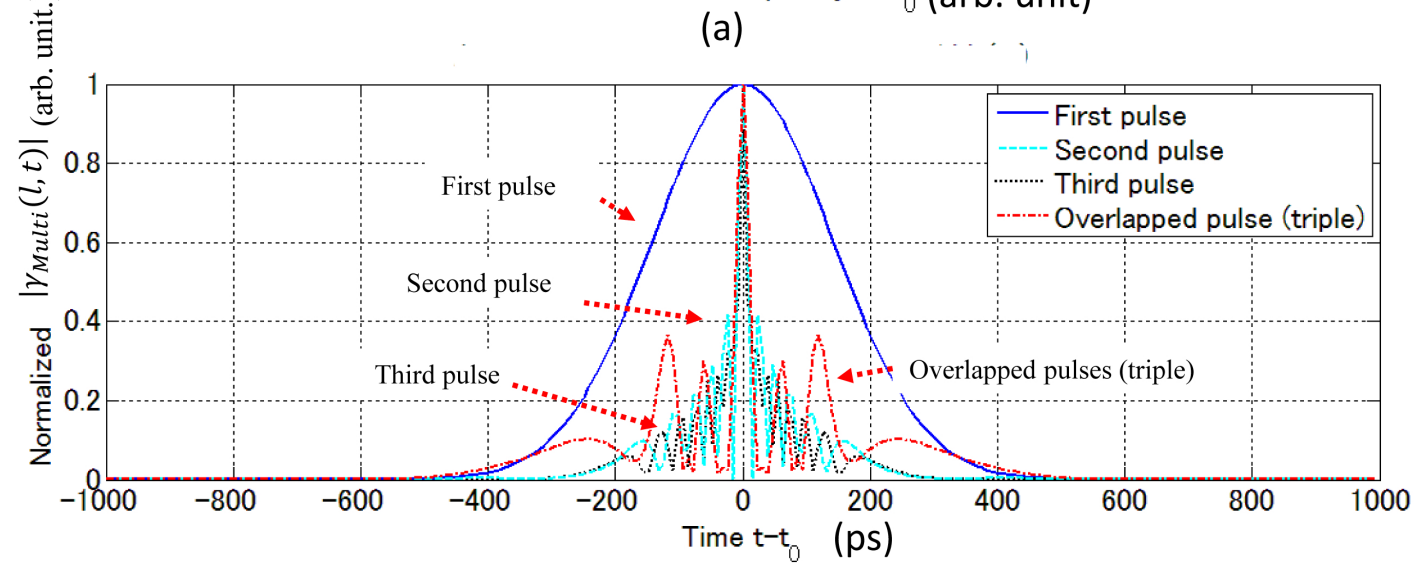

(b)

FIG. 4 TCF shaping by combining three pulses. (a) Spectra of the two propagated pulses, and the result of overlapping the three pulses. (b) Autocorrelation of the initial pulse, the propagated pulses, and the overlap of the three pulses. Due to interference between the three spectra, the autocorrelation function has been transformed into a discrete peak. All three of the overlapped pulses have the same intensity. The maximum phase shifts of the three pulses are $0,10.5 \pi$ (the second pulse), and $14.5 \pi$ (the third pulse), respectively.

proposed method. It is easy to obtain control over the amounts of SPM applied in this technique because we can change the length of the nonlinear fiber by using a piezoelectric element.

\section{SUMMARY}

To conclude, motivated by Fourier series expansion, we researched the possibility of controlling the TCF by overlapping the spectra of different pulses, where the set of modes we chose from were obtained by propagating pulses along different optical path lengths, yielding different amounts of SPM. To the best of our knowledge, this is the first report that indicates that destructive interference between pulses with different amounts of SPM can generate a TCF profile containing distinct peaks. The results of this investigation show that, with the appropriate pulse powers and optical path lengths, the necessary SPM modes can be generated, and combining the resulting pulses can produces a unique TCF. Finally, it is anticipated that this multicomponent SPM-based TCF shaping will be a powerful tool for imaging and metrology.

\section{ACKN OWLEDGEMENTS}

This research work was financially supported by a grant from The Asahi Glass Foundation.

\section{References}

[1] D. J. Frantzeskakis and T. Sphicopoulos, "Nonlinear self-phase modulation in optical soliton systems with lumped amplifiers," Opt. Commun. 101, 337-341 (1993).

[2] R. R. Alfano, The supercontinuum laser source : fundamentals with updated references (Second Edition, Springer, New York, 2006).

[3] S. Larochelle, Y. Hibino, V. Mizrahi, and G. I. Stegeman, "All-optical switching of grating transmission using cross-phase modulation in optical fibres," Electron. Lett. 26, 1459-1460 (1990).

[4] G. P. Agrawal, Nonlinear fiber optics, 4th ed., Quantum electronicsprinciples and applications (Elsevier / Academic Press, Amsterdam/Boston, 2007).

[5] M. Ohta, M. Kuramoto, M. Ikeda, and H. Yokoyama, "Extreme Nonlinear Phase Shift in Self-Pulsating Laser Diodes," in OSA Technical Digest (CD) (Optical Society of America, 2007).

[6] R. H. Stolen and C. Lin, "Self-phase-modulation in silica optical fibers," Phys. Rev. A 17, 1448-1453 (1978).

[7] Y. Zhang, M. Sato, and N. Tanno, "Resolution improvement in optical coherence tomography by optimal synthesis of light-emitting diodes," Opt. Lett. 26, 205-207 (2001).

[8] J. Gong, B. Liu, Y. L. Kim, Y. Liu, X. Li, and V. Backman, “Optimal spectral reshaping for resolution improvement in optical coherence tomography," Opt. Express 14, 5909-5915 (2006).

[9] M. Enyama, and K. Hotate, "Dynamic and random-access strain measurement by fiber Bragg gratings with synthesis of optical coherence function," Proc. SPIE 5589, 144-153 (2004). 
[10] M. Takeda, W. Wang, Z. Duan, and Y. Miyamoto, "Coherence holography," Opt. Express 13, 9629-9635 (2005).

[11] Z. Duan, Y. Miyamoto, and M. Takeda, "Dispersion-free absolute interferometry based on angular spectrum scanning," Opt. Express 14, 655-663 (2006).

[12] Z. Duan, Y. Miyamoto, and M. Takeda, "Dispersion-free optical coherence depth sensing with a spatial frequency comb generated by an angular spectrum modulator," Opt. Express 14, 12109-12121 (2006).

[13] M. Sato, I. Wakaki, Y. Watanabe, and N. Tanno, "Fundamental characteristics of a synthesized light source for optical coherence tomography," Appl. Optics 44, 2471-2481 (2005).

[14] K. Hotate, and Z. He, "Synthesis of Optical-Coherence Function and Its Applications in Distributed and Multiplexed Optical Sensing," J. Lightwave Technol. 24, 2541 (2006).

[15] M. Takeda, W. Wang, and D. Naik, "Coherence Holography: A Thought on Synthesis and Analysis of Optical Coherence Fields," in Fringe 2009, W. Osten and M. Kujawinska, eds., 1-8 (Springer, Berlin Heidelberg, 2009).
[16] T. R. Taha, and M. I. Ablowitz, "Analytical and numerical aspects of certain nonlinear evolution equations. II. Numerical, nonlinear Schrödinger equation," J. Comput. Phys. 55, 203-230 (1984).

[17] 0. V. Sinkin, R. Holzlöhner, J. Zweck, and C. R. Menyuk, "Optimization of the Split-Step Fourier Method in Modeling Optical-Fiber Communications Systems," J. Lightwave Technol. 21, 61 (2003).

[18] D. M. Nguyen, S. D. Le, G. Gueguen, D. Méchin, M. Thual, and T. Chartier, "Numerical method for simultaneous measurement of dispersion and nonlinear coefficient in optical fibers," Opt. Commun. 285, 1461-1465 (2012).

[19] D. Wei, S. Takahashi, K. Takamasu, and H. Matsumoto, "Theoretical Analysis of Length Measurement Using Interference of Multiple Pulse Trains of a Femtosecond Optical Frequency Comb," Jpn. J. Appl. Phys. 50, 022701 (2011).

[20] T. Tanabe, H. Tanabe, Y. Teramura, and F. Kannari, "Spatiotemporal measurements based on spatial spectral interferometry for ultrashort optical pulses shaped by a Fourier pulse shaper," J. Opt. Soc. Am. B 19, 2795-2802 (2002). 